\title{
New integrable systems of derivative nonlinear Schrödinger equations with multiple components
}

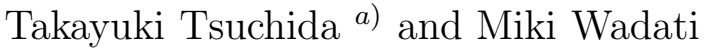 \\ Department of Physics, Graduate School of Science, University of Tokyo, \\ Hongo 7-3-1, Bunkyo-ku, Tokyo 113-0033, Japan
}

(Received 25 June 1998, revised version received 05 January 1999)

The Lax pair for a derivative nonlinear Schrödinger equation proposed by Chen-Lee-Liu is generalized into matrix form. This gives new types of integrable coupled derivative nonlinear Schrödinger equations. By virtue of a gauge transformation, a new multi-component extension of a derivative nonlinear Schrödinger equation proposed by Kaup-Newell is also obtained.

PACS numbers: 02.30.Jr, 03.40.Kf, 42.50.Rh, 42.81.Dp

keywords: derivative nonlinear Schrödinger equation, multi-component system, Lax pair, AKNS formulation, gauge transformation, conservation laws

${ }^{a)}$ Electronic mail: tsuchida@monet.phys.s.u-tokyo.ac.jp 


\section{Introduction}

One of the most remarkable discoveries in soliton theory is the inverse scattering method (ISM). It is well-known that there are two types of derivative nonlinear Schrödinger (DNLS) equation whose complete integrability can be proved by the ISM. One type was proposed by Kaup and Newell [1],

$$
\begin{aligned}
& \mathrm{i} \phi_{t}+\phi_{x x}-\mathrm{i}(\phi \psi \phi)_{x}=0, \\
& \mathrm{i} \psi_{t}-\psi_{x x}-\mathrm{i}(\psi \phi \psi)_{x}=0 .
\end{aligned}
$$

In what follows, we call this system the Kaup-Newell equation for convenience. The other type,

$$
\begin{aligned}
& \mathrm{i} q_{t}+q_{x x}-\mathrm{i} q r q_{x}=0, \\
& \mathrm{i} r_{t}-r_{x x}-\mathrm{i} r q r_{x}=0,
\end{aligned}
$$

was studied by Chen, Lee and Liu [2], who gave its recursion operator. We call this system the Chen-Lee-Liu equation. There is a simple transformation of dependent variables which cast one into the other [3, 4]. In this sense, these two types of the DNLS equation are gauge equivalent.

The ISM provides us a powerful tool to find multi-component extensions of one-component soliton equations [5] such as the nonlinear Schrödinger (NLS) equation [6, 7], the modified $\mathrm{KdV}$ equation 8, 9], the Ablowitz-Ladik system [10, 11]. For the Kaup-Newell equation, it has been known that there is a simple vector generalization [12, 13],

$$
\mathrm{i} \boldsymbol{q}_{t}+\boldsymbol{q}_{x x} \mp \mathrm{i}\left(|\boldsymbol{q}|^{2} \boldsymbol{q}\right)_{x}=\mathbf{0}
$$

which are completely integrable. Besides the system (1.3), Fordy 13 investigated various coupled versions of the Kaup-Newell equation by considering Hermitian symmetric spaces. Yajima studied a generalization of coupled DNLS equations by means of a gauge transformation in [14]. Meanwhile, multi-component extensions of the Chen-Lee-Liu equation have not been studied thoroughly from the ISM point of view.

In recent years, multi-field extensions or matrix generalizations of one-component classical integrable systems have been developed considerably by means of various approaches. Svinolupov, Sokolov, Habibullin and Yamilov clarified close connections between soliton equations and Jordan algebras or Jordan triple systems [15, 16, 17, 18]. An excellent feature of their theory lies in the point that their approach exhausts all integrable cases in some classes of multi-field equations. Olver and Sokolov [19 surveyed integrable systems whose dependent variables take their value in an associative algebra, e.g., matrix-valued systems. They listed some classes of evolution equations on associative algebras which have higher-order symmetries.

In this Letter, we introduce a novel Lax formulation to get a matrix generalization of the Chen-Lee-Liu equation. As reductions of the matrix equation, we obtain two types of coupled Chen-Lee-Liu equations. Through a transformation of variables, one type is cast into the vector Kaup-Newell system (1.3). It is noteworthy that the other type is transformed into a new type of coupled Kaup-Newell equations. The latter type of the coupled Chen-Lee-Liu 
equations is shown to be connected with the coupled NLS equations,

$$
\begin{aligned}
& \mathrm{i} u_{j, t}+u_{j, x x}-2 \sum_{k=1}^{m} u_{k} v_{k} \cdot u_{j}=0 \\
& \mathrm{i} v_{j, t}-v_{j, x x}+2 \sum_{k=1}^{m} v_{k} u_{k} \cdot v_{j}=0
\end{aligned}
$$

This indicates that the new coupled Chen-Lee-Liu equations and the new coupled KaupNewell equations are solvable by the ISM.

The present Letter consists of the following. In section 2, we introduce a new matrix generalization of the Lax pair for the Chen-Lee-Liu equation. Under some reductions, we obtain two types of coupled Chen-Lee-Liu equations. In addition, a systematic method to construct conservation laws is given. In section 3, we perform gauge transformations to the coupled Chen-Lee-Liu equations found in the previous section. A connection between the coupled Chen-Lee-Liu equations and the coupled NLS equations is clarified. In section 4, we propose transformations of variables to consider generalizations of the coupled Chen-LeeLiu equations. With a particular choice of parameters, we obtain a new type of coupled Kaup-Newell equations. The last section, section 5, is devoted to the concluding remarks.

\section{Coupled DNLS equations}

In this section, we consider a matrix generalization of the Chen-Lee-Liu equation by use of the ISM formulation. As reductions, we derive two new integrable systems of coupled Chen-Lee-Liu equations.

\subsection{Lax formulation}

Let us begin with a system of linear equations,

$$
\Psi_{x}=U \Psi, \quad \Psi_{t}=V \Psi .
$$

The compatibility condition $\Psi_{x t}=\Psi_{t x}$ is satisfied if

$$
U_{t}-V_{x}+U V-V U=O .
$$

We call $U, V$ the Lax pair and (2.2) the Lax equation or the zero-curvature condition. We choose the following form of the Lax pair,

$$
\begin{aligned}
& U=\mathrm{i} \zeta^{2}\left[\begin{array}{ll}
-I_{1} & \\
& I_{2}
\end{array}\right]+\zeta\left[\begin{array}{ll} 
& Q \\
R &
\end{array}\right]+\mathrm{i}\left[\begin{array}{cc}
O & \\
& \frac{1}{2} R Q
\end{array}\right], \\
& V=\mathrm{i} \zeta^{4}\left[\begin{array}{ll}
-2 I_{1} & \\
& 2 I_{2}
\end{array}\right]+\zeta^{3}\left[\begin{array}{ll}
2 R & 2 Q \\
2 R &
\end{array}\right]+\mathrm{i} \zeta^{2}\left[\begin{array}{ll}
-Q R & \\
& R Q
\end{array}\right] \\
& +\zeta\left[\begin{array}{lr}
-\mathrm{i} R_{x}+\frac{1}{2} R Q R & \mathrm{i} Q_{x}+\frac{1}{2} Q R Q
\end{array}\right]+\mathrm{i}\left[\begin{array}{ll}
O & \\
& \frac{\mathrm{i}}{2}\left(R Q_{x}-R_{x} Q\right)+\frac{1}{4} R Q R Q
\end{array}\right] .
\end{aligned}
$$


Here, $\zeta$ is the spectral parameter. $I_{1}$ and $I_{2}$ are respectively the $p \times p$ and the $q \times q$ identity matrices. $Q$ is a $p \times q$ matrix and $R$ is a $q \times p$ matrix. Substituting (2.3) and (2.4) into (2.2) and equating the terms with the same powers of $\zeta$, we get a set of nonlinear evolution equations,

$$
\begin{aligned}
& \mathrm{i} Q_{t}+Q_{x x}-\mathrm{i} Q R Q_{x}=O, \\
& \mathrm{i} R_{t}-R_{x x}-\mathrm{i} R_{x} Q R=O .
\end{aligned}
$$

Comments are in order. First, the equation obtained in the order $O\left(\zeta^{0}=1\right)$ of (2.2) is automatically satisfied because of (2.5). Thus, we have no restrictions on the sizes of $Q$ and $R$, that is, on $p$ and $q$. This fact enables us to consider various multi-field extensions of the Chen-Lee-Liu equation by choosing the forms of $Q$ and $R$ appropriately. Second, the trace of $U, \operatorname{tr} U$, depends on dynamical variables in this formulation. Third, Olver and Sokolov showed that the matrix equation (2.5) possess at least one higher symmetry [19], which leads to a conjecture for the integrability of (2.5). The existence of the Lax pair gives a definite support to the complete integrability of the model.

\subsection{Coupled Chen-Lee-Liu equations (type I)}

As a reduction of (2.5), we choose $Q$ and $R$ to be a row vector and a column vector respectively,

$$
Q=\left(q_{1}, q_{2}, \cdots, q_{m}\right), \quad R=\left(r_{1}, r_{2}, \cdots, r_{m}\right)^{T} .
$$

Here the superscript $T$ stands for the transposition. Then, we obtain a coupled version of the Chen-Lee-Liu equation,

$$
\begin{aligned}
& \mathrm{i} q_{j, t}+q_{j, x x}-\mathrm{i} \sum_{k=1}^{m} q_{k} r_{k} \cdot q_{j, x}=0, \\
& \mathrm{i} r_{j, t}-r_{j, x x}-\mathrm{i} \sum_{k=1}^{m} r_{k} q_{k} \cdot r_{j, x}=0,
\end{aligned}
$$

In the following, we call this system the coupled Chen-Lee-Liu I equations or, simply, the type I equations. The explicit form of the Lax matrix $U$ is given by

$$
U=\left[\begin{array}{ccccc}
-\mathrm{i} \zeta^{2} & \zeta q_{1} & \zeta q_{2} & \cdots & \zeta q_{m} \\
\zeta r_{1} & \mathrm{i} \zeta^{2}+\mathrm{i} \frac{1}{2} r_{1} q_{1} & \mathrm{i} \frac{1}{2} r_{1} q_{2} & \cdots & \mathrm{i} \frac{1}{2} r_{1} q_{m} \\
\zeta r_{2} & \mathrm{i} \frac{1}{2} r_{2} q_{1} & \mathrm{i} \zeta^{2}+\mathrm{i} \frac{1}{2} r_{2} q_{2} & \cdots & \mathrm{i} \frac{1}{2} r_{2} q_{m} \\
\vdots & \vdots & \vdots & \ddots & \vdots \\
\zeta r_{m} & \mathrm{i} \frac{1}{2} r_{m} q_{1} & \mathrm{i} \frac{1}{2} r_{m} q_{2} & \cdots & \mathrm{i} \zeta^{2}+\mathrm{i} \frac{1}{2} r_{m} q_{m}
\end{array}\right] .
$$

Under the reduction,

$$
r_{j}= \pm q_{j}^{*}, \quad j=1,2, \cdots, m
$$

the system (2.7) is expressed in a compact form,

$$
\mathrm{i} \boldsymbol{q}_{t}+\boldsymbol{q}_{x x} \mp \mathrm{i}|\boldsymbol{q}|^{2} \boldsymbol{q}_{x}=\mathbf{0},
$$

with $\boldsymbol{q}$ being the vector,

$$
\boldsymbol{q}=\left(q_{1}, q_{2}, \cdots, q_{m}\right) .
$$




\subsection{Conservation laws (type I)}

We can construct an infinite number of conservation laws from the zero-curvature condition and the Lax pair. If we set

$$
\Psi=\left(v_{1}, v_{2}, \cdots, v_{m+1}\right)^{T}
$$

we have

$$
\left(\sum_{j=1}^{m+1} U_{i j} v_{j} v_{i}^{-1}\right)_{t}=\left(\sum_{j=1}^{m+1} V_{i j} v_{j} v_{i}^{-1}\right)_{x}
$$

and

$$
\left(U_{j j}-U_{i i}\right) v_{j} v_{i}^{-1}=-\sum_{k(\neq j)} U_{j k} v_{k} v_{i}^{-1}+\left(v_{j} v_{i}^{-1}\right)_{x}+v_{j} v_{i}^{-1} \sum_{k(\neq i)} U_{i k} v_{k} v_{i}^{-1},
$$

by virtue of (2.1) and (2.2). Introducing a new set of variables $\left\{\Gamma_{j}\right\}$ by

$$
\Gamma_{j} \equiv v_{j+1} v_{1}^{-1}, \quad j=1,2, \cdots, m,
$$

we get from (2.13) and (2.14) with (2.8),

$$
\left(\sum_{j=1}^{m} q_{j} \Gamma_{j}\right)_{t}=\left(\zeta^{-1} V_{11}+\zeta^{-1} \sum_{j=1}^{m} V_{1 j+1} \Gamma_{j}\right)_{x}
$$

and

$$
q_{j} \Gamma_{j}=-\frac{1}{2 \mathrm{i} \zeta} q_{j} r_{j}+\frac{2 \mathrm{i}}{(2 \mathrm{i} \zeta)^{2}} q_{j} \Gamma_{j, x}+\frac{q_{j} r_{j}}{(2 \mathrm{i} \zeta)^{2}} \sum_{k=1}^{m} q_{k} \Gamma_{k}+\frac{1}{2 \mathrm{i} \zeta} q_{j} \Gamma_{j} \sum_{k=1}^{m} q_{k} \Gamma_{k} .
$$

(2.16) shows that $\sum_{j} q_{j} \Gamma_{j}$ is a generating function of conserved densities. We expand $q_{j} \Gamma_{j}$ as

$$
q_{j} \Gamma_{j} \equiv \sum_{l=1}^{\infty} \frac{1}{(2 \mathrm{i} \zeta)^{2 l-1}} f_{j}^{(l)}
$$

to get recursion formulas for the conserved densities,

$$
f_{j}^{(l)}=-q_{j} r_{j} \delta_{l, 1}+2 \mathrm{i} q_{j}\left(q_{j}^{-1} f_{j}^{(l-1)}\right)_{x}+\sum_{n=2}^{l-1} f_{j}^{(n)} \sum_{k=1}^{m} f_{k}^{(l-n)} .
$$

For example, the followings are the first five conserved densities for the coupled Chen-Lee-Liu I equations (2.7),

$$
\begin{array}{ll}
I_{1} & =\sum_{j} q_{j} r_{j}, \\
I_{2} & =q_{j} r_{l, x}, \quad \forall j, l, \\
I_{3} & =-4 \sum_{j} q_{j, x} r_{j, x}+\mathrm{i} \sum_{j}\left(q_{j} r_{j, x}-q_{j, x} r_{j}\right) \cdot \sum_{k} q_{k} r_{k}, \\
I_{4} & =-4 \mathrm{i} \sum_{j}\left(q_{j, x} r_{j, x x}-q_{j, x x} r_{j, x}\right)+8 \sum_{j} q_{j, x} r_{j, x} \sum_{k} q_{k} r_{k}-\left\{\sum_{j}\left(q_{j} r_{j, x}-q_{j, x} r_{j}\right)\right\}^{2}+\left\{\left(\sum_{j} q_{j} r_{j}\right)_{x}\right\}^{2}
\end{array}
$$




$$
\begin{aligned}
& -\mathrm{i} \sum_{j}\left(q_{j} r_{j, x}-q_{j, x} r_{j}\right) \cdot\left(\sum_{k} q_{k} r_{k}\right)^{2}, \\
I_{5}= & -16 \sum_{j} q_{j, x x} r_{j, x x}+12 \mathrm{i} \sum_{j}\left(q_{j, x} r_{j, x x}-q_{j, x x} r_{j, x}\right) \cdot \sum_{k} q_{k} r_{k}-4 \mathrm{i} \sum_{j}\left(q_{j} r_{j, x}-q_{j, x} r_{j}\right) \cdot\left(\sum_{k} q_{k} r_{k}\right)_{x x} \\
& +12 \mathrm{i} \sum_{j} q_{j, x} r_{j, x} \cdot \sum_{k}\left(q_{k} r_{k, x}-q_{k, x} r_{k}\right)-12 \sum_{j} q_{j, x} r_{j, x} \cdot\left(\sum_{k} q_{k} r_{k}\right)^{2}-3 \sum_{j} q_{j} r_{j} \cdot\left\{\left(\sum_{k} q_{k} r_{k}\right)_{x}\right\}^{2} \\
& +3\left\{\sum_{j}\left(q_{j} r_{j, x}-q_{j, x} r_{j}\right)\right\}^{2} \cdot \sum_{k} q_{k} r_{k}+\mathrm{i} \sum_{j}\left(q_{j} r_{j, x}-q_{j, x} r_{j}\right) \cdot\left(\sum_{k} q_{k} r_{k}\right)^{3} .
\end{aligned}
$$

\subsection{Coupled Chen-Lee-Liu equations (type II)}

As another reduction of (2.5), we choose $Q$ and $R$ to be a column vector and a row vector respectively,

$$
Q=\left(\tilde{q}_{1}, \tilde{q}_{2}, \cdots, \tilde{q}_{m}\right)^{T}, \quad R=\left(\tilde{r}_{1}, \tilde{r}_{2}, \cdots, \tilde{r}_{m}\right) .
$$

In this case, (2.5) reduces to

$$
\begin{aligned}
& \mathrm{i} \tilde{q}_{j, t}+\tilde{q}_{j, x x}-\mathrm{i} \sum_{k=1}^{m} \tilde{q}_{k, x} \tilde{r}_{k} \cdot \tilde{q}_{j}=0, \\
& \mathrm{i} \tilde{r}_{j, t}-\tilde{r}_{j, x x}-\mathrm{i} \sum_{k=1}^{m} \tilde{r}_{k, x} \tilde{q}_{k} \cdot \tilde{r}_{j}=0,
\end{aligned}
$$

We call this system the coupled Chen-Lee-Liu II equations or, simply, the type II equations in what follows. Hisakado proposed the coupled Chen-Lee-Liu II equations independently in [20]. The Lax matrix $U$ for (2.22) is given by

$$
U=\left[\begin{array}{cccc}
-\mathrm{i} \zeta^{2} & & & \zeta \tilde{q}_{1} \\
& \ddots & \vdots \\
& & -\mathrm{i} \zeta^{2} & \zeta \tilde{q}_{m} \\
\zeta \tilde{r}_{1} & \cdots & \zeta \tilde{r}_{m} & \mathrm{i} \zeta^{2}+\mathrm{i} \frac{1}{2} \sum_{k=1}^{m} \tilde{r}_{k} \tilde{q}_{k}
\end{array}\right]
$$

The representation of the Lax pair is not unique. We can employ a different representation of $U$ for (2.22) as

$$
U=\left[\begin{array}{ccccc}
-\mathrm{i} \zeta^{2}-\mathrm{i} \frac{1}{2} \sum_{k=1}^{m} & \tilde{q}_{k} \tilde{r}_{k} & \zeta \tilde{q}_{1} & \cdots & \zeta \tilde{q}_{m} \\
\zeta \tilde{r}_{1} & \mathrm{i} \zeta^{2} & & \\
\vdots & & \ddots & \\
\zeta \tilde{r}_{m} & & & \mathrm{i} \zeta^{2}
\end{array}\right]
$$

The difference in the structure of the Lax matrices (2.8) and (2.23) (or (2.24)) should be noteworthy. We can calculate an infinite set of conservation laws for (2.22) in the same manner as in section 2.3. The recursion relations for the conserved densities are

$$
g_{j}^{(l)}=-q_{j} r_{j} \delta_{l, 1}+2 \mathrm{i} q_{j}\left(q_{j}^{-1} g_{j}^{(l-1)}\right)_{x}+\sum_{n=1}^{l-2} g_{j}^{(n)} \sum_{k=1}^{m} g_{k}^{(l-n)},
$$


which yield an infinite series of conserved densities $\sum_{l} g_{j}^{(l)}$. The first five conserved densities are, for instance,

$$
\begin{aligned}
\tilde{I}_{1}= & \sum_{j} \tilde{q}_{j} \tilde{r}_{j}, \\
\tilde{I}_{2}= & \mathrm{i} \sum_{j}\left(\tilde{q}_{j} \tilde{r}_{j, x}-\tilde{q}_{j, x} \tilde{r}_{j}\right) \\
\tilde{I}_{3}= & -4 \sum_{j} \tilde{q}_{j, x} \tilde{r}_{j, x}+\mathrm{i} \sum_{j}\left(\tilde{q}_{j} \tilde{r}_{j, x}-\tilde{q}_{j, x} \tilde{r}_{j}\right) \cdot \sum_{k} \tilde{q}_{k} \tilde{r}_{k}, \\
\tilde{I}_{4}= & -4 \mathrm{i} \sum_{j}\left(\tilde{q}_{j, x} \tilde{r}_{j, x x}-\tilde{q}_{j, x x} \tilde{r}_{j, x}\right)+4 \sum_{j} \tilde{q}_{j, x} \tilde{r}_{j, x} \sum_{k} \tilde{q}_{k} \tilde{r}_{k}-2\left\{\sum_{j}\left(\tilde{q}_{j} \tilde{r}_{j, x}-\tilde{q}_{j, x} \tilde{r}_{j}\right)\right\}^{2}+2\left\{\left(\sum_{j} \tilde{q}_{j} \tilde{r}_{j}\right)_{x}\right\}^{2} \\
& -\mathrm{i} \sum_{j}\left(\tilde{q}_{j} \tilde{r}_{j, x}-\tilde{q}_{j, x} \tilde{r}_{j}\right) \cdot\left(\sum_{k} \tilde{q}_{k} \tilde{r}_{k}\right)^{2}, \\
\tilde{I}_{5}= & -16 \sum_{j} \tilde{q}_{j, x x} \tilde{r}_{j, x x}+4 \mathrm{i} \sum_{j}\left(\tilde{q}_{j, x} \tilde{r}_{j, x x}-\tilde{q}_{j, x x} \tilde{r}_{j, x}\right) \cdot \sum_{k} \tilde{q}_{k} \tilde{r}_{k}-12 \mathrm{i} \sum_{j}\left(\tilde{q}_{j} \tilde{r}_{j, x}-\tilde{q}_{j, x} \tilde{r}_{j}\right) \cdot\left(\sum_{k} \tilde{q}_{k} \tilde{r}_{k}\right)_{x x} \\
& +20 \mathrm{i} \sum_{j} \tilde{q}_{j, x} \tilde{r}_{j, x} \cdot \sum_{k}\left(\tilde{q}_{k} \tilde{r}_{k, x}-\tilde{q}_{k, x} \tilde{r}_{k}\right)-4 \sum_{j} \tilde{q}_{j, x} \tilde{r}_{j, x} \cdot\left(\sum_{k} \tilde{q}_{k} \tilde{r}_{k}\right)^{2}-5 \sum_{j} \tilde{q}_{j} \tilde{r}_{j} \cdot\left\{\left(\sum_{k} \tilde{q}_{k} \tilde{r}_{k}\right)_{x}\right\}^{2} \\
& +5\left\{\sum_{j}\left(\tilde{q}_{j} \tilde{r}_{j, x}-\tilde{q}_{j, x} \tilde{r}_{j}\right)\right\}^{2} \cdot \sum_{k} \tilde{q}_{k} \tilde{r}_{k}+\mathrm{i} \sum_{j}\left(\tilde{q}_{j} \tilde{r}_{j, x}-\tilde{q}_{j, x} \tilde{r}_{j}\right) \cdot\left(\sum_{k} \tilde{q}_{k} \tilde{r}_{k}\right)^{3} \cdot
\end{aligned}
$$

It is interesting to compare (2.20b) and (2.26b): $\tilde{I}_{2}$ is a conserved density only after taking a summation with respect to the subscript $j$.

\section{Gauge transformations}

In this section, we investigate the structure of the Lax pairs given in the previous section in connection with the AKNS formulation [21]. For this purpose, we introduce a gauge transformation [3],

$$
\Psi=g \Phi
$$

which changes the Lax matrices $U, V$ into

$$
\begin{aligned}
& U^{\prime}=g^{-1} U g-g^{-1} g_{x}, \\
& V^{\prime}=g^{-1} V g-g^{-1} g_{t} .
\end{aligned}
$$

In the following, dependent variables are assumed to approach 0 as $|x| \rightarrow \infty$ for convenience.

\section{$3.1 \quad$ type I}

In terms of $\left\{q_{j}\right\},\left\{r_{j}\right\}$ which satisfy (2.7), we introduce a new set of variables $\left\{u_{j}\right\},\left\{v_{j}\right\}$ by

$$
\begin{aligned}
& u_{j}=a q_{j} \exp \left\{-\frac{\mathrm{i}}{2} \int_{-\infty}^{x} \sum_{k=1}^{m} q_{k} r_{k} \mathrm{~d} x^{\prime}\right\}, \\
& v_{j}=b r_{j, x} \exp \left\{\frac{\mathrm{i}}{2} \int_{-\infty}^{x} \sum_{k=1}^{m} q_{k} r_{k} \mathrm{~d} x^{\prime}\right\},
\end{aligned}
$$


Here the constants $a$ and $b$ satisfy $a b=-\mathrm{i} / 2$. Using (3.3) and the first conservation law for (2.7), we obtain

$$
\begin{aligned}
& \mathrm{i} u_{j, t}+u_{j, x x}-2 \sum_{k=1}^{m} u_{k} v_{k} \cdot u_{j}=\left[\mathrm{i} q_{j, t}+q_{j, x x}-\mathrm{i} \sum_{k=1}^{m} q_{k} r_{k} \cdot q_{j, x}\right] a \exp \left\{-\frac{\mathrm{i}}{2} \int_{-\infty}^{x} \sum_{k=1}^{m} q_{k} r_{k} \mathrm{~d} x^{\prime}\right\}, \\
& \mathrm{i} v_{j, t}-v_{j, x x}+2 \sum_{k=1}^{m} v_{k} u_{k} \cdot v_{j}=\left[\mathrm{i} r_{j, t}-r_{j, x x}-\mathrm{i} \sum_{k=1}^{m} r_{k} q_{k} \cdot r_{j, x}\right] b \exp \left\{\frac{\mathrm{i}}{2} \int_{-\infty}^{x} \sum_{k=1}^{m} q_{k} r_{k} \mathrm{~d} x^{\prime}\right\} .
\end{aligned}
$$

Hence, we conclude that if $\left\{q_{j}\right\}$ and $\left\{r_{j}\right\}$ satisfy the coupled Chen-Lee-Liu I equations, $\left\{u_{j}\right\}$ and $\left\{v_{j}\right\}$ satisfy the well-known coupled NLS equations,

$$
\begin{aligned}
& \mathrm{i} u_{j, t}+u_{j, x x}-2 \sum_{k=1}^{m} u_{k} v_{k} \cdot u_{j}=0 \\
& \mathrm{i} v_{j, t}-v_{j, x x}+2 \sum_{k=1}^{m} v_{k} u_{k} \cdot v_{j}=0
\end{aligned}
$$

By means of the transformation (3.3), the second conserved densities (2.20b) are cast into the second conserved densities for (3.5),

$$
I_{2}^{\prime}=u_{j} v_{l}, \quad \forall j, l .
$$

The Lax matrix for the coupled NLS equations (3.5) is obtained from (2.8) through a gauge transformation,

$$
g=\left[\begin{array}{cccc}
2 \mathrm{i} b \zeta \mathrm{e}^{K} & 0 & \cdots & 0 \\
-b r_{1} \mathrm{e}^{K} & 1 & & \\
\vdots & & \ddots & \\
-b r_{m} \mathrm{e}^{K} & & & 1
\end{array}\right], \quad K=\frac{\mathrm{i}}{2} \int_{-\infty}^{x} \sum_{k=1}^{m} q_{k} r_{k} \mathrm{~d} x^{\prime}
$$

as

$$
\begin{aligned}
U^{\prime} & =g^{-1} U g-g^{-1} g_{x} \\
& =\left[\begin{array}{cccc}
-\mathrm{i} \zeta^{2} & u_{1} & \cdots & u_{m} \\
v_{1} & \mathrm{i} \zeta^{2} & & \\
\vdots & & \ddots & \\
v_{m} & & & \mathrm{i} \zeta^{2}
\end{array}\right]
\end{aligned}
$$

We thus have shown that the scattering problem for the coupled Chen-Lee-Liu I equations associated with (2.8) is gauge equivalent to $s l(m+1)$ AKNS formulation. 


\section{2 type II}

Next, let us consider a gauge transformation of the Lax matrix for the coupled Chen-Lee-Liu II equations. By virtue of a gauge transformation,

$$
g=\left[\begin{array}{cccc}
2 \mathrm{i} b \zeta & & & \\
& \ddots & & \\
& & 2 \mathrm{i} b \zeta & \\
-b \tilde{r}_{1} & \cdots & -b \tilde{r}_{m} & 1
\end{array}\right]
$$

the Lax matrix $(2.23)$ is cast into

$$
\begin{aligned}
& U^{\prime}=g^{-1} U g-g^{-1} g_{x} \\
& =\left[\begin{array}{cccc}
-\mathrm{i} \zeta^{2} & & & \\
& \ddots & & \\
& & -\mathrm{i} \zeta^{2} & \\
& & & \mathrm{i} \zeta^{2}
\end{array}\right]+\left[\begin{array}{cccc}
\mathrm{i} \frac{1}{2} \tilde{q}_{1} \tilde{r}_{1} & \cdots & \mathrm{i} \frac{1}{2} \tilde{q}_{1} \tilde{r}_{m} & a \tilde{q}_{1} \\
\vdots & \ddots & \vdots & \vdots \\
\mathrm{i} \frac{1}{2} \tilde{q}_{m} \tilde{r}_{1} & \cdots & \mathrm{i} \frac{1}{2} \tilde{q}_{m} \tilde{r}_{m} & a \tilde{q}_{m} \\
b \tilde{r}_{1, x} & \cdots & b \tilde{r}_{m, x} & 0
\end{array}\right],
\end{aligned}
$$

with $a b=-\mathrm{i} / 2$. It is noticed that there is no longer the spectral parameter $\zeta$ in the potential part of $U^{\prime}$. We can easily eliminate dependent variables in the diagonal elements of $U^{\prime}$ by a further transformation. Thus, the gauge-transformed Lax formulation is embedded in the $(m+1) \times(m+1)$ matrix generalization of the AKNS formulation 22, 23].

\section{Generalizations}

In this section, we consider generalizations of the coupled Chen-Lee-Liu equations (type I \& II) via transformations of variables.

\section{1 type I}

We first transform the coupled Chen-Lee-Liu I equations into a system with cubic terms without differentiation. Let us change independent and dependent variables by

$$
\begin{array}{r}
T=t, \quad X=x+\frac{2 \beta}{\alpha} t, \\
q_{j}=\sqrt{\alpha} Q_{j} \exp \left\{-\mathrm{i} \frac{\beta}{\alpha} X+\mathrm{i}\left(\frac{\beta}{\alpha}\right)^{2} T\right\}, \\
r_{j}=\sqrt{\alpha} R_{j} \exp \left\{\mathrm{i} \frac{\beta}{\alpha} X-\mathrm{i}\left(\frac{\beta}{\alpha}\right)^{2} T\right\},
\end{array}
$$

From (2.7), we get a system of equations,

$$
\begin{aligned}
& \mathrm{i} Q_{j, T}+Q_{j, X X}-\mathrm{i} \alpha \sum_{k=1}^{m} Q_{k} R_{k} \cdot Q_{j, X}-\beta \sum_{k=1}^{m} Q_{k} R_{k} \cdot Q_{j}=0, \\
& \mathrm{i} R_{j, T}-R_{j, X X}-\mathrm{i} \alpha \sum_{k=1}^{m} R_{k} Q_{k} \cdot R_{j, X}+\beta \sum_{k=1}^{m} R_{k} Q_{k} \cdot R_{j}=0,
\end{aligned}
$$


We further utilize a kind of gauge transformation,

$$
\begin{aligned}
& \phi_{j}=Q_{j} \exp \left\{-2 \mathrm{i} \delta \int_{-\infty}^{X} \sum_{k=1}^{m} Q_{k} R_{k} \mathrm{~d} X^{\prime}\right\}, \\
& \psi_{j}=R_{j} \exp \left\{2 \mathrm{i} \delta \int_{-\infty}^{X} \sum_{k=1}^{m} Q_{k} R_{k} \mathrm{~d} X^{\prime}\right\},
\end{aligned}
$$

and get an extension of (2.7),

$$
\begin{aligned}
& \mathrm{i} \phi_{j, T}+\phi_{j, X X}-\beta \sum_{k=1}^{m} \phi_{k} \psi_{k} \cdot \phi_{j}+4 \mathrm{i} \delta \sum_{k=1}^{m} \phi_{k} \psi_{k, X} \cdot \phi_{j} \\
& +\mathrm{i}(4 \delta-\alpha) \sum_{k=1}^{m} \phi_{k} \psi_{k} \cdot \phi_{j, X}+\delta(4 \delta+\alpha)\left(\sum_{k=1}^{m} \phi_{k} \psi_{k}\right)^{2} \phi_{j}=0, \\
& \mathrm{i} \psi_{j, T}-\psi_{j, X X}+\beta \sum_{k=1}^{m} \psi_{k} \phi_{k} \cdot \psi_{j}+4 \mathrm{i} \delta \sum_{k=1}^{m} \psi_{k} \phi_{k, X} \cdot \psi_{j} \\
& +\mathrm{i}(4 \delta-\alpha) \sum_{k=1}^{m} \psi_{k} \phi_{k} \cdot \psi_{j, X}-\delta(4 \delta+\alpha)\left(\sum_{k=1}^{m} \psi_{k} \phi_{k}\right)^{2} \psi_{j}=0,2, \cdots, m .
\end{aligned}
$$

For a choice of $4 \delta+\alpha=0$, the system reads

$$
\begin{aligned}
& \mathrm{i} \phi_{j, T}+\phi_{j, X X}-\mathrm{i} \alpha \sum_{k=1}^{m} \phi_{k} \psi_{k, X} \cdot \phi_{j}-2 \mathrm{i} \alpha \sum_{k=1}^{m} \phi_{k} \psi_{k} \cdot \phi_{j, X}-\beta \sum_{k=1}^{m} \phi_{k} \psi_{k} \cdot \phi_{j}=0 \\
& \mathrm{i} \psi_{j, T}-\psi_{j, X X}-\mathrm{i} \alpha \sum_{k=1}^{m} \psi_{k} \phi_{k, X} \cdot \psi_{j}-2 \mathrm{i} \alpha \sum_{k=1}^{m} \psi_{k} \phi_{k} \cdot \psi_{j, X}+\beta \sum_{k=1}^{m} \psi_{k} \phi_{k} \cdot \psi_{j}=0
\end{aligned}
$$

For $m=1$, this is equivalent to the Kaup-Newell equation with a cubic term [24]. Thus, the system (4.6) is interpreted as a new multi-field extension of the Kaup-Newell equation. We can give the explicit expression of the corresponding Lax pair. For instance, the Lax matrix for (4.6) with $\alpha=1, \beta=0$ is given by

$$
U=\left[\begin{array}{ccccc}
-\mathrm{i} \zeta^{2}+\mathrm{i} \frac{1}{2} \sum_{k=1}^{m} \phi_{k} \psi_{k} & \zeta \phi_{1} & \zeta \phi_{2} & \cdots & \zeta \phi_{m} \\
\zeta \psi_{1} & \mathrm{i} \zeta^{2}+\mathrm{i} \frac{1}{2} \psi_{1} \phi_{1} & \mathrm{i} \frac{1}{2} \psi_{1} \phi_{2} & \cdots & \mathrm{i} \frac{1}{2} \psi_{1} \phi_{m} \\
\zeta \psi_{2} & \mathrm{i} \frac{1}{2} \psi_{2} \phi_{1} & \mathrm{i} \zeta^{2}+\mathrm{i} \frac{1}{2} \psi_{2} \phi_{2} & \cdots & \mathrm{i} \frac{1}{2} \psi_{2} \phi_{m} \\
\vdots & \vdots & \vdots & \ddots & \vdots \\
\zeta \psi_{m} & \mathrm{i} \frac{1}{2} \psi_{m} \phi_{1} & \mathrm{i} \frac{1}{2} \psi_{m} \phi_{2} & \cdots & \mathrm{i} \zeta^{2}+\mathrm{i} \frac{1}{2} \psi_{m} \phi_{m}
\end{array}\right]
$$

\section{2 type II}

Following the same procedure as type I, we obtain a generalization of the coupled Chen-LeeLiu II equations (2.22). By a change of variables,

$$
T=t, \quad X=x+\frac{2 \beta}{\alpha} t
$$




$$
\begin{aligned}
& \tilde{q}_{j}=\sqrt{\alpha} \tilde{Q}_{j} \exp \left\{-\mathrm{i} \frac{\beta}{\alpha} X+\mathrm{i}\left(\frac{\beta}{\alpha}\right)^{2} T\right\}, \\
& \tilde{r}_{j}=\sqrt{\alpha} \tilde{R}_{j} \exp \left\{\mathrm{i} \frac{\beta}{\alpha} X-\mathrm{i}\left(\frac{\beta}{\alpha}\right)^{2} T\right\},
\end{aligned}
$$

we get a system of equations,

$$
\begin{aligned}
& \mathrm{i} \tilde{Q}_{j, T}+\tilde{Q}_{j, X X}-\mathrm{i} \alpha \sum_{k=1}^{m} \tilde{Q}_{k, X} \tilde{R}_{k} \cdot \tilde{Q}_{j}-\beta \sum_{k=1}^{m} \tilde{Q}_{k} \tilde{R}_{k} \cdot \tilde{Q}_{j}=0 \\
& \mathrm{i} \tilde{R}_{j, T}-\tilde{R}_{j, X X}-\mathrm{i} \alpha \sum_{k=1}^{m} \tilde{R}_{k, X} \tilde{Q}_{k} \cdot \tilde{R}_{j}+\beta \sum_{k=1}^{m} \tilde{R}_{k} \tilde{Q}_{k} \cdot \tilde{R}_{j}=0
\end{aligned}
$$

By virtue of a gauge transformation,

$$
\begin{aligned}
& \tilde{\phi}_{j}=\tilde{Q}_{j} \exp \left\{-2 \mathrm{i} \delta \int_{-\infty}^{X} \sum_{k=1}^{m} \tilde{Q}_{k} \tilde{R}_{k} \mathrm{~d} X^{\prime}\right\}, \\
& \tilde{\psi}_{j}=\tilde{R}_{j} \exp \left\{2 \mathrm{i} \delta \int_{-\infty}^{X} \sum_{k=1}^{m} \tilde{Q}_{k} \tilde{R}_{k} \mathrm{~d} X^{\prime}\right\}
\end{aligned}
$$

we obtain

$$
\begin{aligned}
& \mathrm{i} \tilde{\phi}_{j, T}+\tilde{\phi}_{j, X X}-\beta \sum_{k=1}^{m} \tilde{\phi}_{k} \tilde{\psi}_{k} \cdot \tilde{\phi}_{j}-\mathrm{i} \alpha \sum_{k=1}^{m} \tilde{\phi}_{k, X} \tilde{\psi}_{k} \cdot \tilde{\phi}_{j}+4 \mathrm{i} \delta \sum_{k=1}^{m} \tilde{\phi}_{k} \tilde{\psi}_{k, X} \cdot \tilde{\phi}_{j} \\
& +4 \mathrm{i} \delta \sum_{k=1}^{m} \tilde{\phi}_{k} \tilde{\psi}_{k} \cdot \tilde{\phi}_{j, X}+\delta(4 \delta+\alpha)\left(\sum_{k=1}^{m} \tilde{\phi}_{k} \tilde{\psi}_{k}\right)^{2} \tilde{\phi}_{j}=0, \\
& \mathrm{i} \tilde{\psi}_{j, T}-\tilde{\psi}_{j, X X}+\beta \sum_{k=1}^{m} \tilde{\psi}_{k} \tilde{\phi}_{k} \cdot \tilde{\psi}_{j}-\mathrm{i} \alpha \sum_{k=1}^{m} \tilde{\psi}_{k, X} \tilde{\phi}_{k} \cdot \tilde{\psi}_{j}+4 \mathrm{i} \delta \sum_{k=1}^{m} \tilde{\psi}_{k} \tilde{\phi}_{k, X} \cdot \tilde{\psi}_{j} \\
& +4 \mathrm{i} \delta \sum_{k=1}^{m} \tilde{\psi}_{k} \tilde{\phi}_{k} \cdot \tilde{\psi}_{j, X}-\delta(4 \delta+\alpha)\left(\sum_{k=1}^{m} \tilde{\psi}_{k} \tilde{\phi}_{k}\right)^{2} \tilde{\psi}_{j}=0,
\end{aligned}
$$

In case of $4 \delta+\alpha=0$, the system essentially reduces to the well-known vector Kaup-Newell system (1.3) in a slightly different notation,

$$
\begin{aligned}
& \mathrm{i} \tilde{\phi}_{j, T}+\tilde{\phi}_{j, X X}-\mathrm{i} \alpha\left(\sum_{k=1}^{m} \tilde{\phi}_{k} \tilde{\psi}_{k} \cdot \tilde{\phi}_{j}\right)_{X}-\beta \sum_{k=1}^{m} \tilde{\phi}_{k} \tilde{\psi}_{k} \cdot \tilde{\phi}_{j}=0, \\
& \mathrm{i} \tilde{\psi}_{j, T}-\tilde{\psi}_{j, X X}-\mathrm{i} \alpha\left(\sum_{k=1}^{m} \tilde{\psi}_{k} \tilde{\phi}_{k} \cdot \tilde{\psi}_{j}\right)_{X}+\beta \sum_{k=1}^{m} \tilde{\psi}_{k} \tilde{\phi}_{k} \cdot \tilde{\psi}_{j}=0,
\end{aligned}
$$

This shows that the coupled Chen-Lee-Liu II equations are gauge equivalent to the vector Kaup-Newell system. The Lax matrix for (4.13) with $\alpha=1, \beta=0$ is given by [7, 12]

$$
U=\left[\begin{array}{cccc}
-\mathrm{i} \zeta^{2} & & & \zeta \tilde{\phi}_{1} \\
& \ddots & & \vdots \\
& & -\mathrm{i} \zeta^{2} & \zeta \tilde{\phi}_{m} \\
\zeta \tilde{\psi}_{1} & \cdots & \zeta \tilde{\psi}_{m} & \mathrm{i} \zeta^{2}
\end{array}\right]
$$

This can also be derived from (2.23) by means of a gauge transformation. 


\section{Concluding remarks}

In this Letter, we have presented a matrix formulation of the inverse scattering method (ISM) and have found the two types of the coupled Chen-Lee-Liu equations, (2.7) and (2.22). As is often the case with one-component soliton systems [7, 13, 15, 16, 17, 18, 19], the Chen-Lee-Liu equation also has plural multi-field generalizations. Using the Lax pairs, the conservation laws and the gauge transformations, we have studied the properties of the two types of the coupled Chen-Lee-Liu equations. An important step to obtain the coupled derivative NLS equations in our theory is the introduction of $U$ by (2.3). The form of $U$ seems unusual, because we more or less assume tr $U$ to be a constant (often equal to 0 ) for soliton systems. The Lax pair is, however, transformed into the well-known one for the coupled NLS equations $(s l(m+1)$ AKNS formulation), which is solvable by the ISM. The ISM for the coupled Chen-Lee-Liu I equations (2.7) will be reported in detail in a separate paper.

In the two-component case, the coupled Chen-Lee-Liu equations (type I \& II) and the related models maybe have physical significances. They may describe wave propagations in birefringent optical fibers with nonlinear effects such as the Raman scattering and the Kerr effect.

The coupled Chen-Lee-Liu equations reported in this Letter have an infinite number of conservation laws and are completely integrable. We can construct other flows of the hierarchies by employing corresponding time-dependences of the scattering problems $(\zeta$-dependences of the Lax matrix $V$ ). These flows have in common the conserved densities for the original flows. Expanding the matrix $V$ from $O\left(\zeta^{6}\right)$ to $O(1)$, we get

$$
\begin{aligned}
& q_{j, t}+\frac{1}{2} q_{j, x x x}-\mathrm{i} \frac{3}{4}\left(\sum_{k} q_{k, x} r_{k} q_{j, x}+\sum_{k} q_{k} r_{k} q_{j, x x}\right)-\frac{3}{8}\left(\sum_{k} q_{k} r_{k}\right)^{2} q_{j, x}=0, \\
& r_{j, t}+\frac{1}{2} r_{j, x x x}+\mathrm{i} \frac{3}{4}\left(\sum_{k} r_{k, x} q_{k} r_{j, x}+\sum_{k} r_{k} q_{k} r_{j, x x}\right)-\frac{3}{8}\left(\sum_{k} r_{k} q_{k}\right)^{2} r_{j, x}=0,
\end{aligned}
$$

for the coupled Chen-Lee-Liu I hierarchy (cf. (2.8)), and

$$
\begin{aligned}
& \tilde{q}_{j, t}+\frac{1}{2} \tilde{q}_{j, x x x}-\mathrm{i} \frac{3}{4}\left(\sum_{k} \tilde{q}_{k, x} \tilde{r}_{k} \tilde{q}_{j, x}+\sum_{k} \tilde{q}_{k, x x} \tilde{r}_{k} \tilde{q}_{j}\right)-\frac{3}{8} \sum_{k} \tilde{q}_{k} \tilde{r}_{k} \sum_{l} \tilde{q}_{l, x} \tilde{r}_{l} \tilde{q}_{j}=0, \\
& \tilde{r}_{j, t}+\frac{1}{2} \tilde{r}_{j, x x x}+\mathrm{i} \frac{3}{4}\left(\sum_{k} \tilde{r}_{k, x} \tilde{q}_{k} \tilde{r}_{j, x}+\sum_{k} \tilde{r}_{k, x x} \tilde{q}_{k} \tilde{r}_{j}\right)-\frac{3}{8} \sum_{k} \tilde{r}_{k} \tilde{q}_{k} \sum_{l} \tilde{r}_{l, x} \tilde{q}_{l} \tilde{r}_{j}=0,
\end{aligned}
$$

for the coupled Chen-Lee-Liu II hierarchy (cf. (2.23)).

On the other hand, by expanding $V$ from $O\left(\zeta^{-2}\right)$ to $O(1)$, we obtain coupled versions of 
the massive Thirring model in a light-cone frame [25],

$$
\begin{aligned}
& q_{j, t}-2 \mathrm{i} \varphi_{j}-\mathrm{i} \frac{1}{2} \sum_{k} \varphi_{k} \sigma_{k} q_{j}=0 \\
& r_{j, t}+2 \mathrm{i} \sigma_{j}+\mathrm{i} \frac{1}{2} \sum_{k} \sigma_{k} \varphi_{k} r_{j}=0 \\
& \varphi_{j, x}+2 \mathrm{i} q_{j}+\mathrm{i} \frac{1}{2} \sum_{k} \varphi_{k} r_{k} q_{j}=0 \\
& \sigma_{j, x}-2 \mathrm{i} r_{j}-\mathrm{i} \frac{1}{2} \sum_{k} \sigma_{k} q_{k} r_{j}=0
\end{aligned}
$$

for the coupled Chen-Lee-Liu I hierarchy, and

$$
\begin{aligned}
& \tilde{q}_{j, t}-2 \mathrm{i} \tilde{\varphi}_{j}-\mathrm{i} \frac{1}{2} \sum_{k} \tilde{q}_{k} \tilde{\sigma}_{k} \tilde{\varphi}_{j}=0 \\
& \tilde{r}_{j, t}+2 \mathrm{i} \tilde{\sigma}_{j}+\mathrm{i} \frac{1}{2} \sum_{k} \tilde{r}_{k} \tilde{\varphi}_{k} \tilde{\sigma}_{j}=0 \\
& \tilde{\varphi}_{j, x}+2 \mathrm{i} \tilde{q}_{j}+\mathrm{i} \frac{1}{2} \sum_{k} \tilde{q}_{k} \tilde{r}_{k} \tilde{\varphi}_{j}=0 \\
& \tilde{\sigma}_{j, x}-2 \mathrm{i} \tilde{r}_{j}-\mathrm{i} \frac{1}{2} \sum_{k} \tilde{r}_{k} \tilde{q}_{k} \tilde{\sigma}_{j}=0
\end{aligned}
$$

for the coupled Chen-Lee-Liu II hierarchy. Here, the explicit form of $V$ is expressed as

$$
V=\mathrm{i} \zeta^{-2}\left[\begin{array}{cc}
I & \\
& -I
\end{array}\right]+\zeta^{-1}\left[\begin{array}{ll} 
& \varphi \\
\sigma &
\end{array}\right]+\mathrm{i}\left[\begin{array}{ll}
\frac{1}{2} \varphi \sigma & \\
& O
\end{array}\right] .
$$

$\varphi_{j}\left(\tilde{\varphi}_{j}\right)$ and $\sigma_{j}\left(\tilde{\sigma}_{j}\right)$ are components of a row (column) vector $\varphi$ and a column (row) vector $\sigma$ for the system (5.3) (5.4)) respectively. By a simple change of variables such as $t \leftrightarrow x$, (5.3) and (5.4) are mutually transformed into the other. The split into two different systems is not essential at the level of these flows. 


\section{References}

[1] D. J. Kaup and A. C. Newell, J. Math. Phys. 19 (1978) 798.

[2] H. H. Chen, Y. C. Lee and C. S. Liu, Phys. Scr. 20 (1979) 490.

[3] M. Wadati and K. Sogo, J. Phys. Soc. Japan 52 (1983) 394.

[4] A. Kundu, J. Math. Phys. 25 (1984) 3433.

[5] M. J. Ablowitz and P. A. Clarkson, Solitons, Nonlinear Evolution Equations and Inverse Scattering (Cambridge University Press, Cambridge, 1991).

[6] S. V. Manakov, Sov. Phys. JETP 38 (1974) 248.

[7] A. P. Fordy and P. P. Kulish, Commun. Math. Phys. 89 (1983) 427.

[8] N. Yajima and M. Oikawa, Prog. Theor. Phys. 54 (1975) 1576.

[9] T. Tsuchida and M. Wadati, J. Phys. Soc. Japan 67 (1998) 1175.

[10] T. Tsuchida, H. Ujino and M. Wadati, J. Math. Phys. 39 (1998) 4785.

[11] T. Tsuchida, H. Ujino and M. Wadati, J. Phys. A 32 (1999) 2239.

[12] H. C. Morris and R. K. Dodd, Phys. Scr. 20 (1979) 505.

[13] A. P. Fordy, J. Phys. A 17 (1984) 1235.

[14] T. Yajima, J. Phys. Soc. Japan 64 (1995) 1901.

[15] S. I. Svinolupov, Commun. Math. Phys. 143 (1992) 559.

[16] S. I. Svinolupov, Func. Anal. Appl. 27 (1993) 257.

[17] S. I. Svinolupov and V. V. Sokolov, Theor. Math. Phys. 100 (1994) 959.

[18] I. T. Habibullin, V. V. Sokolov and R. I. Yamilov, Multi-component integrable systems and nonassociative structures, in Nonlinear Physics: Theory and Experiment (World Scientific, Singapore, 1996) 139.

[19] P. J. Olver and V. V. Sokolov, Commun. Math. Phys. 193 (1998) 245.

[20] M. Hisakado, Chiral solitons from dimensional reduction of Chern-Simons gauged coupled non-linear Schrödinger model, hep-th/9712255.

[21] M. J. Ablowitz, D. J. Kaup, A. C. Newell, and H. Segur, Phys. Rev. Lett. 31 (1973) 125.

[22] V. E. Zakharov and A. B. Shabat, Func. Anal. Appl. 8 (1974) 226. 
[23] M. J. Ablowitz and R. Haberman, J. Math. Phys. 16 (1975) 230.

[24] M. Wadati, K. Konno and Y. Ichikawa, J. Phys. Soc. Japan 46 (1979) 1965.

[25] T. Tsuchida and M. Wadati, J. Phys. Soc. Japan 65 (1996) 3153. 